\title{
Ocorrência de lepidópteros de importância médica (Lepidoptera: Aididae, Lasiocampidae, Limacodidae e Megalopygidae) no Cerro da Buena, município de Morro Redondo, Rio Grande do Sul, Brasil
}

\author{
Ricardo Russo Siewert ${ }^{1 *}$ \\ Eduardo José Ely e Silva ${ }^{2}$ \\ ${ }^{1}$ PPG em Biologia Animal, Departamento de Zoologia, Universidade Federal do Rio Grande do Sul \\ Avenida Bento Gonçalves, 9500, Prédio 43435, Laboratório 218 \\ CEP 91501-970 Porto Alegre - RS, Brasil \\ ${ }^{2}$ Museu Entomológico Ceslau Biezanko, Departamento de Fitossanidade \\ Faculdade de Agronomia Eliseu Maciel, Universidade Federal de Pelotas, Campus Universitário, s/n., \\ Caixa Postal 354, CEP 96010-900, Pelotas, RS, Brasil \\ *Autor para correspondência \\ ricardo_siewert@yahoo.com.br
}

Submetido em 01/02/2012

Aceito para publicação em 29/08/2012

\section{Resumo}

O propósito deste estudo foi registrar a fauna de lepidópteros de importância médica ocorrente no Cerro da Buena, município de Morro Redondo, Rio Grande do Sul, Brasil, entre fevereiro de 2009 a janeiro de 2011. Foram registradas 32 espécies de mariposas, pertencentes à Aididae (1), Lasiocampidae (17), Limacodidae (7) e Megalopygidae (7). Três novas ocorrências de Lasiocampidae para o Rio Grande do Sul foram realizadas: Artace argentina Schaus, 1924, Euglyphis kotzschi Draudt, 1927 e Tolype trilinea (Dognin, 1901).

Palavras-chave: Inventário; Mariposas; Sul do Brasil

\section{Abstract}

Occurrence of lepidopterans of medical importance (Lepidoptera: Aididae, Lasiocampidae, Limacodidae e Megalopygidae) in the Cerro da Buena, Morro Redondo municipality, Rio Grande do Sul, Brazil. This study recorded the lepidopterans of medical importance from Cerro da Buena in Rio Grande do Sul State. Field expeditions were carried out from February 2009 to January 2011. Thirty-two moth species were recorded that were distributed in the Aididae (1), Lasiocampidae (17), Limacodidae (7) and Megalopygidae (7). Three new records of Lasiocampidae are reported for Rio Grande do Sul: Artace argentina Schaus, 1924, Euglyphis kotzschi Draudt, 1927 and Tolype trilinea (Dognin, 1901).

Key words: Inventory; Moths; Southern Brazil 
Entre os insetos de importância médica, algumas espécies de lepidópteros destacam-se por apresentar estruturas capazes de produzir e inocular substâncias de ação urticante. $O$ contato direto com a fase imatura destes lepidópteros pode causar acidentes como dermatites urticantes, periartrite falangeana e até síndrome hemorrágica (e.g. CARDOSO; JUNIOR, 2005; SPECHT et al., 2008).

Objetivando documentar os registros de lepidópteros de importância médica ocorrentes no Rio Grande do Sul, o projeto denominado 'Lepidópteros de Importância Médica no Rio Grande do Sul' listou 160 espécies ocorrentes (SPECHT et al., 2004; 2005a; 2005b; 2006). Entretanto, esta listagem foi gerada através de revisões bibliográficas e exames em coleções científicas, não contemplando informações precisas sobre os locais de registros. Os autores sugerem que maiores esforços de coletas sejam efetivados para que se obtenham mais informações biológicas e ecológicas destas espécies.
Com o intuito de contribuir para o conhecimento da fauna de lepidópteros de importância médica do Rio Grande do Sul, o presente estudo inventariou a fauna de mariposas das famílias Aididae, Lasiocampidae, Limacodidade e Megalopygidae ocorrentes no Cerro da Buena ( $31^{\circ} 43^{\prime} \mathrm{S}$ e $52^{\circ} 38^{\prime} \mathrm{W}, 245 \mathrm{~m}$ altitude), localizado no município de Morro Redondo (Figura 1), inserido no Bioma Pampa, região sul do Estado. O clima na região é do tipo temperado $(\mathrm{Cfb})$ com temperatura anual de $16,7^{\circ} \mathrm{C}$ e precipitação média anual de $1400 \mathrm{~mm}$ (KUINCHTNER; BURIOL, 2001). A vegetação ocorrente no local é composta por um mosaico de Floresta Estacional Semidecidual e Estepe arborizada (CORDEIRO; HASENACK, 2009), circundado por plantações de milho, feijão, pastagens e soja.

As coletas foram realizadas durante os meses de fevereiro de 2009 a janeiro de 2011, sempre no período novilúnio, uma vez ao mês, totalizando 24 amostragens. As mariposas foram atraídas por uma

FIGURA 1: Mapa do Estado do Rio Grande do Sul indicando a localização (•) do município de Morro Redondo (31³5’18” S, 53³6’54”'W).

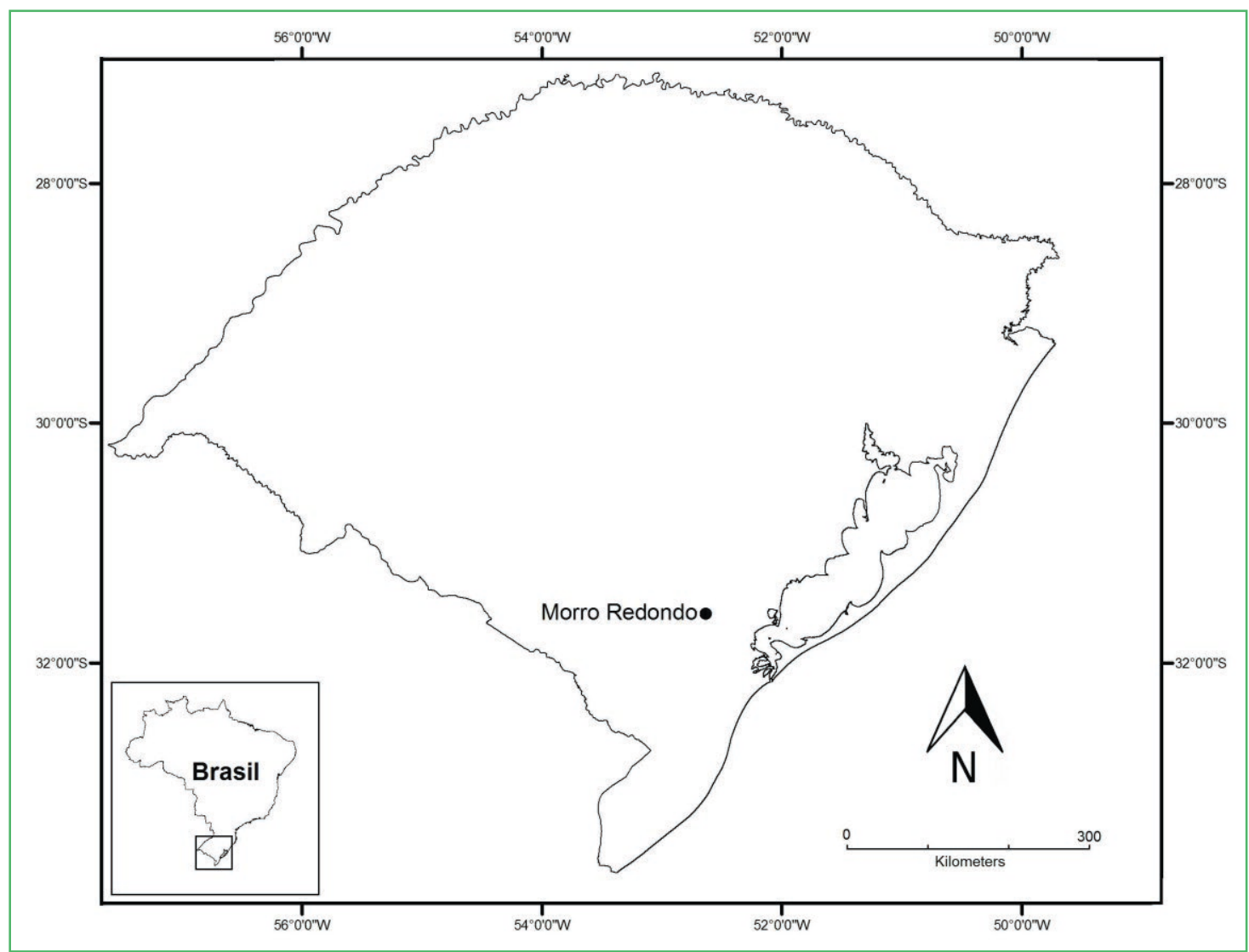


fonte luminosa (lâmpada mista de mercúrio de 250W) posicionada a $1,5 \mathrm{~m}$ do solo e refletida em um tecido branco (2mx2m) estendido (FERRO; DINIZ, 2007). A armadilha foi ligada ao anoitecer e permaneceu até a manhã do dia seguinte. Os indivíduos foram capturados manualmente e sacrificados com o auxílio de frascos mortíferos contendo acetato de etila. Posteriormente, foram levados ao laboratório para distensão, secagem em estufa e identificação. O material testemunho encontra-se depositado no Museu Entomológico Ceslau Biezanko (MECB), do Departamento de Fitossanidade da Faculdade de Agronomia Eliseu Maciel da Universidade Federal de Pelotas.

Estimou-se a riqueza de mariposas ocorrentes no Cerro da Buena empregando os estimadores não paramétricos Chao 2 e Jackknife 2, pois, de acordo com Colwell e Coddington (1994) e Magurran (2004), estes levam em consideração apenas a presença e ausência das espécies entre os conjuntos de dados e são mais confiáveis quando se tem um número pequeno de amostras. As análises das estimativas de riqueza foram realizadas no ambiente de programação $R$ version 2.13.2, utilizando os pacotes 'vegan' e 'fossil' (R DEVELOPMENT CORE TEAM, 2011).

Foram registrados 188 indivíduos, pertencentes a 32 espécies de mariposas, distribuídas em Aididae, Lasiocampidae, Limacodidae e Megalopygidae (Tabela 1).

Três espécies constituem novas ocorrências para o

TABELA 1: Lepidópteros de importância médica (Lepidoptera: Aididae, Lasiocampidae, Limacodidae e Megalopygidae) coletados no Cerro da Buena, município de Morro Redondo, Rio Grande do Sul, Brasil. (*) Novos registros para o Estado.

\begin{tabular}{|c|c|c|}
\hline Taxa & Abundância & Meses/Anos \\
\hline \multicolumn{3}{|l|}{ Aididae $(S=1)$} \\
\hline Brachycodilla carmen (Schaus, 1892) & 7 & Fev(09/10)/ Mar(09)/ Out(09)/ Nov(10) \\
\hline \multicolumn{3}{|l|}{ Lasiocampidae $(S=17)$} \\
\hline \multicolumn{3}{|l|}{ Macromphaliinae (S=17) } \\
\hline Artace argentina Schaus, 1924 * & 1 & $\operatorname{Abr}(09)$ \\
\hline Artace cribraria (Ljungh, 1825) & 2 & $\operatorname{Dez}(09) / \operatorname{Mai}(10)$ \\
\hline Artace menuve Schaus, 1924 & 3 & $\operatorname{Jan}(10) / \mathrm{Fev}(10) / \operatorname{Mai}(10)$ \\
\hline Artace rubripalpis (C. Felder, 1874) & 9 & Fev(09/10)/Out(09)/ Abr(10)/ Mai(10)/Jan(11) \\
\hline Euglyphis deusta (Herrich-Schäffer, [1854]) & 9 & $\operatorname{Fev}(09 / 10) / \operatorname{Mar}(09) / \operatorname{Nov}(09) / \operatorname{Abr}(10) / \operatorname{Dez}(10)$ \\
\hline Euglyphis fibra (Schaus, 1890) & 3 & Out $(09) /$ Fev $(10)$ \\
\hline Euglyphis guttularis (Walker, 1855) & 2 & Mai(09) \\
\hline Euglyphis kotzschi Draudt, $1927 *$ & 3 & Abr(09)/ Mai(09)/ Ago(10) \\
\hline Euglyphis lacrimosa (Schaus, 1892) & 5 & $\operatorname{Mar}(09) / \operatorname{Jan}(10) / \operatorname{Nov}(10) / \operatorname{Dez}(10)$ \\
\hline Euglyphis plana (Walker, 1855) & 12 & $\operatorname{Mar}(09 / 10) / \operatorname{Mai}(09) / \operatorname{Out}(09) / \operatorname{Nov}(09 / 10) / \operatorname{Dez}(09)$ \\
\hline Euglyphis semifunebris (Schaus, 1915) & 5 & $\operatorname{Jun}(09) / \operatorname{Jul}(09 / 10) / \operatorname{Ago}(09)$ \\
\hline Euglyphis submarginalis (Walker, 1866) & 2 & Fev(10)/Mai(10) \\
\hline Titya alma (Weymer, 1895) & 6 & $\operatorname{Fev}(09) / \operatorname{Mar}(09)$ \\
\hline Titya trilinea (Dognin, 1901) * & 1 & $\operatorname{Jan}(10)$ \\
\hline Tolype indecisa (Walker, 1855) & 7 & $\operatorname{Fev}(09) / \operatorname{Mar}(09 / 10) / \operatorname{Abr}(09 / 10)$ \\
\hline Tolype medialis (Jones, 1912) & 2 & $\operatorname{Mai}(10) / \operatorname{Jun}(10)$ \\
\hline Tolype pellita Draudt, 1927 & 3 & $\operatorname{Abr}(10) / \operatorname{Mai}(10)$ \\
\hline \multicolumn{3}{|l|}{ Limacodidae $(S=7)$} \\
\hline Acharia norans (Dyar, 1927) & 1 & $\operatorname{Nov}(09)$ \\
\hline Miresa clarissa (Stoll, 1790) & 3 & $\operatorname{Fev}(09) / \operatorname{Jan}(11)$ \\
\hline Parasa mionexia Dyar, 1924 & 4 & $\operatorname{Fev}(09 / 10) / \operatorname{Jan}(10)$ \\
\hline Perola petropolis Dyar, 1905 & 21 & $\operatorname{Mar}(09)$ \\
\hline Perola subpunctata (Walker, 1855) & 12 & $\operatorname{Mar}(09)$ \\
\hline Talima rufoflava (Walker, 1855) & 27 & $\begin{array}{c}\operatorname{Fev}(09 / 10) / \operatorname{Mar}(09 / 10) / \operatorname{Abr}(09 / 10) / \operatorname{Mai}(09) / \operatorname{Nov}(09 / 10) / \\
\operatorname{Dez}(09 / 10) / \operatorname{Jan}(10)\end{array}$ \\
\hline Talima sp. & 1 & $\operatorname{Mai}(09)$ \\
\hline
\end{tabular}




\begin{tabular}{|c|c|c|}
\hline \multicolumn{3}{|l|}{ Megalopygidae (S=7) } \\
\hline \multicolumn{3}{|l|}{ Megalopyginae ( $S=4)$} \\
\hline Megalopyge amita Schaus, 1900 & 2 & $\operatorname{Jan}(10)$ \\
\hline Podalia albescens (Schaus, 1900) & 2 & $\operatorname{Nov}(10)$ \\
\hline Podalia dimidiata (Herrich-Schäffer, [1856]) & 13 & $\operatorname{Fev}(09 / 10) / \operatorname{Mar}(09) / \operatorname{Jan}(10)$ \\
\hline Podalia walkeri (Berg, 1882) & 1 & $\operatorname{Nov}(09)$ \\
\hline \multicolumn{3}{|l|}{ Trosiinae $(S=3)$} \\
\hline Norape albilineata (Hopp, 1927) & 6 & $\operatorname{Out}(09) / \operatorname{Nov}(09 / 10) / \operatorname{Abr}(10)$ \\
\hline Norape beggoides (Dyar, 1910) & 4 & Set(09)/ Out(09)/ Nov(09) \\
\hline Trosia dimas (Cramer, 1775) & 9 & $\operatorname{Mar}(09 / 10)$ \\
\hline
\end{tabular}

Estado do Rio Grande do Sul: Artace argentina Schaus, 1924, Euglyphis kotzschi Draudt, 1927 e Tolype trilinea (Dognin, 1901), todas de Lasiocampidae. Os imaturos do gênero Artace são facilmente encontrados em espécies do gênero Eryngium (Apiaceae), planta bastante comum na área amostrada (BIEZANKO, 1974). Destaca-se ainda o registro de seis exemplares de Titya alma (Weymer, 1895) (Lasiocampidae) considerada espécie rara no Estado (BIEZANKO, 1983). Entre os Megalopygidae, a espécie Podalia dimidiata (Herrich-Schäffer, [1856]) foi a mais abundante, com 13 indivíduos registrados. Esta mariposa tem como planta-hospedeira o gênero Lithrea (Anacardiaceae) (BIEZANKO, 1974), outra espécie comum na área de estudo. Entre os Limacodidae registrados, Parasa mionexia Dyar, 1924 e Talima rufoflava (Walker, 1855) tinham sua distribuição conhecida até a região das Missões (SPECHT et al. 2005a), distando aproximadamente $510 \mathrm{~km}$ da área do presente estudo.

De acordo com os estimadores, a riqueza estimada foi de 36 e 41 espécies, sugerindo que entre $78 \%$ e $87 \%$ da fauna local de lepidópteros que apresentam algum tipo de importância médica foi registrada. Cabe salientar, que Hemileucinae, um dos principais grupos de lepidópteros com espécies de importância médica, não foi considerado por fazer parte de outro estudo realizado pelos mesmos autores (SIEWERT et al., dados não publicados).

Tendo em vista a escassez de trabalhos envolvendo inventários da fauna de mariposas, ressalta-se a importância de avaliar padrões gerais de ocorrência e distribuição de lepidópteros. Com base neste conhecimento, é possível aplicar uma padronização em protocolos de amostragens que tenham diferentes fins, como por exemplo, inventários faunísticos a curto ou longo prazo com objetivos conservacionistas.

O Cerro da Buena sofre acentuada ação antrópica devido à implementação de monoculturas, as quais se tornam cada vez mais frequentes na região. Apesar disto, os dados obtidos neste estudo possibilitam a ampliação da distribuição de algumas espécies e registros de outras tidas como raras e/ou escassas no Estado, ressaltando a importância da preservação de áreas e fragmentos para a manutenção da biodiversidade local. Aliando isto à escassez de informações acerca destes grupos, sugere-se a intensificação de inventários e estudos sobre a biologia de tais espécies.

\section{Agradecimentos}

Os autores agradecem ao Sr. Alfred Moser, Dr. Vitor O. Becker, Biólogo Anderson Nunes Prestes e Msc. Cintia Lepesqueur Gonçalves pelo auxílio nas identificações de alguns exemplares e pela bibliografia solicitada. Aos dois revisores anônimos pelas críticas e sugestões.

\section{Referências}

BIEZANKO, C. M. Plantas y otras sustâncias alimentícias de las orugas de los lepidópteros uruguayos. Revista do Centro de Ciências Rurais, Santa Maria, v. 4, n. 2, p.107-148, 1974.

BIEZANKO, C. M. Adelocephalidae, Saturniidae, Mimallonidae, Lasiocampidae, Eupterotidae et Lymantriidae da Região Sueste do Rio Grande do Sul. Revista do Centro de Ciências Rurais, Santa Maria, v. 13, n. 2-3, p. 97-121, 1983.

CARDOSO, A. E. C.; JUNIOR, V. H. Acidentes por Lepidópteros (larvas e adultos de mariposas): estudo dos aspectos epidemiológicos, clínicos e terapêuticos. Anais Brasileiros de Dermatologia, Rio de Janeiro, v. 80, n. 6, p. 571-578, 2005. 
COLWELL, R. K.; CODDINGTON, J. A. Estimating terrestrial biodiversity through extrapolation. Philosophical Transactions of the Royal Society of London, Series B, Biological Science, Londres, v. 345, p. 101-118, 1994.

CORDEIRO, J. L. P.; HASENACK, H. 2009. Cobertura vegetal atual do Rio Grande do Sul. In: PILLAR, V. D.; MÜLLER, S. C.; CASTILHOS, Z. M. S.; JACQUES, A. V. A. (Ed.). Campos Sulinos: conservação e uso sustentável da biodiversidade. Brasília: Ministério do Meio Ambiente, 2009. p. 285-299.

FERRO, V. G.; DINIZ, I. R. Composição de espécies de Arctiidae (Lepidoptera) em áreas de Cerrado. Revista Brasileira de Zoologia, Curitiba, v. 24, n. 3, p. 635-646, 2007.

KUINCHTNER, A.; BURIOL, G. A. Clima do Estado do Rio Grande do Sul segundo a classificação climática de Köppen e Thornthwaite. Disciplinarum Scientia, Santa Maria, v. 2, p. 171182, 2001.

MAGURRAN, A. E. Measuring biological diversity. Oxford: Blackwell Science, 2004. 256 p.

$\mathrm{R}$ DEVELOPMENT CORE TEAM. R: A language and environment for statistical computing. Version 2.13.2. 2011. Disponível em: <http://www.R-project.org>. Acesso em: out. 2011. SPECHT, A.; CORSEUIL, E.; ABELLA, H. B. Lepidópteros de importância médica - principais espécies ocorrentes no Rio
Grande do Sul. Pelotas: USEB, 2008. 220 p.

SPECHT, A.; CORSEUIL, E.; FORMENTINI, A. C. Lepidópteros de importância médica ocorrentes no Rio Grande do Sul - II. Aididae e Limacodidae. Biociências, Porto Alegre, v. 13, n. 1, p. 89-94, 2005a.

SPECHT, A.; CORSEUIL, E.; FORMENTINI, A. C. Lepidópteros de importância médica ocorrentes no Rio Grande do Sul - III Saturniidae, Hemileucinae. Biociências, Porto Alegre, v. 13, n. 2, p. 149-162, 2005b.

SPECHT, A.; CORSEUIL, E.; FORMENTINI, A. C. Lepidópteros de importância médica ocorrentes no Rio Grande do Sul - IV Lasiocampidae. Biociências, Porto Alegre, v. 14, n. 1, p. 53-60, 2006.

SPECHT, A.; CORSEUIL, E.; FORMENTINI, A. C; PRESTES, A. $\mathrm{S}$. Lepidópteros de importância médica ocorrentes no Rio Grande do Sul - I. Megalopygidae. Biociências, Porto Alegre, v. 12, n. 2, p. 173-179, 2004.

WOLDA, H. Insect seasonality: Why? Annual Review of Ecology and Systematics, Palo Alto, v. 19, p. 1-18, 1988. 